\title{
Viva bem com a coluna que você tem: ação multidisciplinar no tratamento da lombalgia
}

\author{
Live well with a spine you have: multidisciplinary action on low back \\ pain treatment
}

Daniel Rogério Petreça'; Paula Fabrício Sandreschi; Fabíola Herbst Rodrigues²; Regiane Koaski; Luiz Eduardo Becker; Nilton Furquim Junior'; Giovana Zarpellon Mazo ${ }^{3}$

\begin{abstract}
Resumo
Esse estudo objetiva descrever a implementação do projeto: "Viva bem com a coluna que você tem". O projeto, criado em 2004, com parceria entre a Prefeitura Municipal de Mafra e a Universidade do Contestado, visa promover bem-estar físico, social e diminuição do quadro álgico de indivíduos com lombalgia por meio de tratamento multidisciplinar. Desde a sua criação já foram atendidas cerca de 500 pessoas. O projeto é desenvolvido em uma academia de musculação, duas vezes por semana, com duração de 60 minutos, por meio de exercícios aeróbios, de flexibilidade e resistência de força. A sessão é conduzida por uma equipe composta por fisioterapeutas, profissionais e estudantes de educação física. Os participantes melhoraram/mantiveram a aptidão física relacionada à saúde e diminuíram o quadro álgico. Com isso, o projeto tem atingido seus objetivos podendo ser adotado como uma estratégia alternativa para o tratamento da lombalgia na saúde pública.
\end{abstract}

\section{Palavras-chave}

Dor lombar; Educação Física e Treinamento; Fisioterapia; Atividade Motora.

\begin{abstract}
This study has the goal to describe the implementation of the project: "Live well with a spine you have". This project, beginning in 2004, with the partnership of the City Hall of Mafra and the University of the Contestado, aims to promote the physical and social well being and decrease the pain symptoms of individuals with low back pain by a multidisciplinary treatment and produce new knowledge. Since it's creation 500 people have been attended. The project is developed in a GYM, twice a week, with the duration of 60 minutes, through aerobic exercises, of flexibility and strength resistance. The session is conducted by a team composed of physiotherapists, professionals, and students of physical education. The participants have improved/kept the fitness related to health and decreased the pain symptoms. With that, the project has achieved it's results been able to be adopted as an alternative strategy for the low back pain treatment in public health.

\section{Keywords}

Low back pain; Physical Education and Training; Physical Therapy Specialty; Motor Activity.
\end{abstract}

\section{Introdução}

A dor lombar é uma condição que tem afeta muitos indivíduos ao longo de suas vidas ${ }^{1}$. Atinge principalmente mulheres e sua prevalência aumenta linearmente da terceira década de vida até os 60 anos de idade 2 . Estima-se que $8,8 \%$ das mulheres e $7,2 \%$ dos homens sul americanos apresentam lombalgia ${ }^{3}$; e ao analisar somente o Brasil, 25,4\% da população possui essa condição².

Além dos malefícios individuais, a dor lombar é responsável pelo aumento do afastamento do trabalho

1 Universidade do Contestado, Núcleo de Educação Física, Núcleo de Pesquisa em Saúde Coletiva e Meio Ambiente, Universidade do Contestado, Mafra, Santa Catarina, Brasil

2 Secretaria Municipal da Saúde de Mafra, Programa Mafra em Forma, Mafra, Santa Catarina, Brasil

3 Universidade do Estado de Santa Catarina, Laboratório de Gerontologia, Universidade do Estado de Santa Catarina, Florianópolis, Santa Catarina, Brasil por licenças médicas e dos custos com saúde ${ }^{4,5}$. Nesse sentido, exercícios combinados parecem ser uma alternativa não farmacológica, não cirúrgica e mais barata para a diminuição da dor ${ }^{6}$. Há evidências que tratamentos envolvendo pilates ${ }^{7}$, terapia manual aliada à exercício físico ${ }^{8}$ e diferentes técnicas fisioterapêuticas ${ }^{9}$ são eficazes para que essa condição seja minimizada.

Assim, devido a lombalgia ser uma condição que causa debilidade ${ }^{3}$, a sua elevada prevalência ${ }^{1}$ é fundamental que os setores públicos e privados de saúde, baseado em evidências alternativas eficazes de tratamento, com grupos multidisciplinares de atenção, implementem intervenções a fim de reduzir a alta demanda de atendimento. Assim, esse estudo tem como objetivo descrever a implementação do projeto "Viva 
bem com a coluna que você tem, uma ação multidisciplinar no tratamento da lombalgia", aplicado na cidade de Mafra, Santa Catarina.

\section{Métodos}

\section{Histórico e objetivos do projeto}

Criado em 2004, o projeto multidisciplinar para o tratamento de lombalgia tem como nome fantasia "Viva bem com a coluna que você tem!" inspirado no livro de Knoplich $^{10}$. Seu funcionamento acontece por meio da parceria institucional entre a Prefeitura Municipal de Mafra por intermédio da Secretaria Municipal da Saúde (SMS) e do Programa Mafra em Forma com a Universidade do Contestado (UnC), Campus Mafra pelo Núcleo de Educação Física.

O projeto tem como objetivo atender aos usuários do Sistema Único de Saúde (SUS) com lombalgia. O tratamento é multidisciplinar e envolve procedimentos fisioterapêuticos e exercício físicos a fim de promover bem-estar físico, social e diminuição do quadro álgico. Dessa forma, auxiliando na produção do conhecimento das áreas de Fisioterapia e Educação Física. Já foram atendidos, desde sua implantação, aproximadamente 500 usuários.

\section{Funcionamento}

O projeto possui como público alvo usuários com lombalgia e o ingresso acontece por meio de requisição médica. Ao receber as requisições, a equipe de fisioterapeutas realiza previamente uma avaliação fisioterapêutica na Clínica Municipal de Reabilitação. Caso seja aprovado, o usuário é encaminhado para a avaliação realizada no laboratório de avaliação física e performance humana da Universidade do Contestado. Após liberação dos dois setores, o usuário é incluído no grupo de intervenção. Ocasionalmente, indivíduos com outras condições como: desvios posturais, pós-cirúrgico (osteomuscular) incontinência urinária, entre outros, são incluídos. A inclusão se dá por meio de reunião entre o grupo de trabalho.

São disponibilizadas cerca de 40 vagas em fluxo contínuo. As sessões acontecem de forma coletiva, atendendo de 10 a 12 usuários por horário. Na sua maioria, os participantes são mulheres, acima de 50 anos e tem conhecimento do projeto por meio da divulgação em jornal e por indicação de amigos e parentes.

Anualmente, a parceria entre as instituições se dá por meio da assinatura de convênio de cooperação técnica, científica e educativa, visando o desenvolvimento de projetos em promoção da saúde. A SMS disponibiliza o fisioterapeuta e destina R\$ 5.000,00/ano (cinco mil reais/ano) para manutenção dos equipamentos utilizados na academia. A UnC disponibiliza o corpo docente, o espaço físico e media a seleção dos estagiários e voluntários. O projeto é iniciado após a assinatura do convênio entre a UnC e a SMS. Suas ações são realizadas de acordo com o calendário acadêmico da Universidade seguindo a estrutura de planejamento apresentado na Figura 1.

O projeto é desenvolvido na academia de musculação da UnC localizada no Centro Poliesportivo do Núcleo de Educação Física, Campus Mafra. As sessões de 60 minutos são realizadas duas vezes por semana das $07 \mathrm{~h} 00$ às $11 \mathrm{~h} 00$ por uma equipe composta de um coordenador geral (UnC, 2 horas/semana) um fisioterapeuta (SMS, 8 horas/semana), um profissional de educação física (UnC, 8 horas/ semana) além de dois estagiários (20 horas/semana) e 1 voluntário estudante em Educação Física (8 horas/semana). Os profissionais são indicados pelas instituições de origem e os estagiários passam por processo seletivo. Os indicadores de avaliação são identificados por meio dos dados obtidos na avaliação fisioterápica e na avaliação física, sendo analisados nas reuniões do grupo de trabalho. 


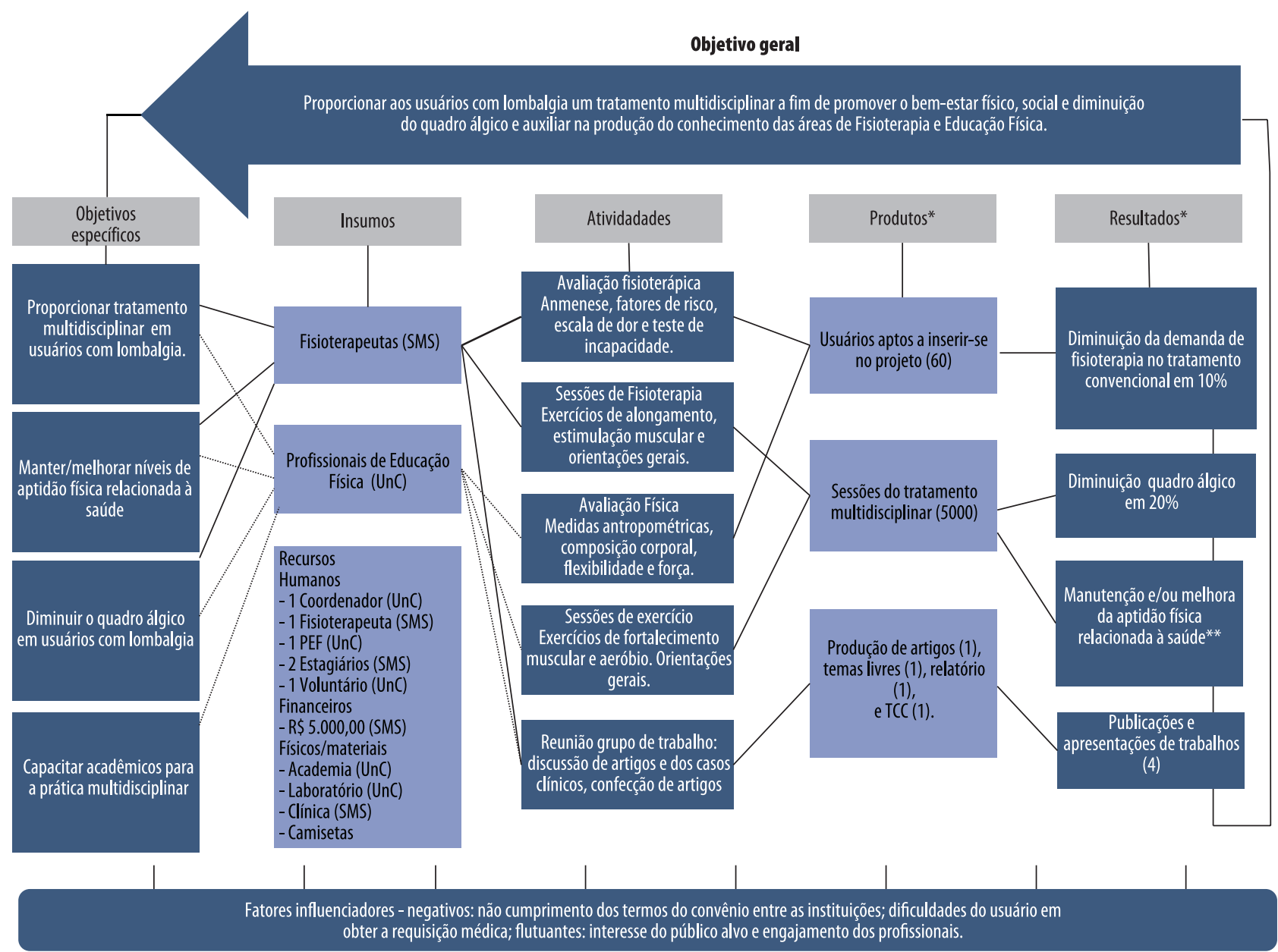

FIGURA 1 - Modelo lógico do projeto: Viva bem com a coluna que você tem!, Mafra, Santa Catarina, Brasil (2016).

UnC: Universidade do Contestado; SMS: Secretaria Municipal da Saúde; PEF: Profissional de educação física; * Produtos e resultados referentes ao ano de 2015; ** A melhora e/ou manutenção da aptidão física relacionada à saúde tem metas individuais de acordo com o quadro inicial do usuário.

\section{Protocolo de tratamento}

Os participantes são submetidos a uma bateria de testes antes do início das sessões de treinamento contendo: ficha diagnóstica (dados sociodemográficos e fatores de risco); Escala de Borg (CR-10) ${ }^{11}$ adaptada para verificação do quadro álgico (Figura 2); questionário de incapacidade de Roland Morris ${ }^{12}$; medidas antropométricas de massa corporal e estatura; Índice de Massa Corporal; composição corporal por meio por meio de bioimpedância elétrica; teste de flexibilidade sentar e alcançar; teste de flexibilidade de ombros; força de preensão manual por meio de dinamômetro.

O protocolo de tratamento acontece na seguinte ordem: exercícios de alongamento para membros inferiores e superiores sendo utilizado cinco movimentos em três séries de 30 segundos em posição decúbito dorsal; após, em pé, cinco movimentos para alongamento dos membros superiores. Posteriormente iniciam-se os exercícios aeróbios, podendo ser aplicados em esteira e/ou bicicleta ergométrica. São realizados de 15 a 20 minutos, sendo a intensidade entre $60 \%$ a $70 \%$ obtidas por subjetividade. Em seguida, inicia-se o treinamento de força contendo 10 movimentos para os grandes grupos musculares em 3 séries de 15 repetições com 40 segundos de intervalo, além de exercícios abdominais. As intensidades são obtidas por meio de esforço subjetivo sendo utilizado entre 60 a $70 \%$ por meio de nota identificada pelo usuário (seis ou sete). O grupo de trabalho prescreve exercícios 
aeróbios e de força adicionais em casos particulares mediante aos dados obtidos com o processo de avaliação inicial. Ainda, os participantes recebem orientação para realização de exercícios domiciliares de flexibilidade e cuidados com a postura principalmente em casa/ trabalho.

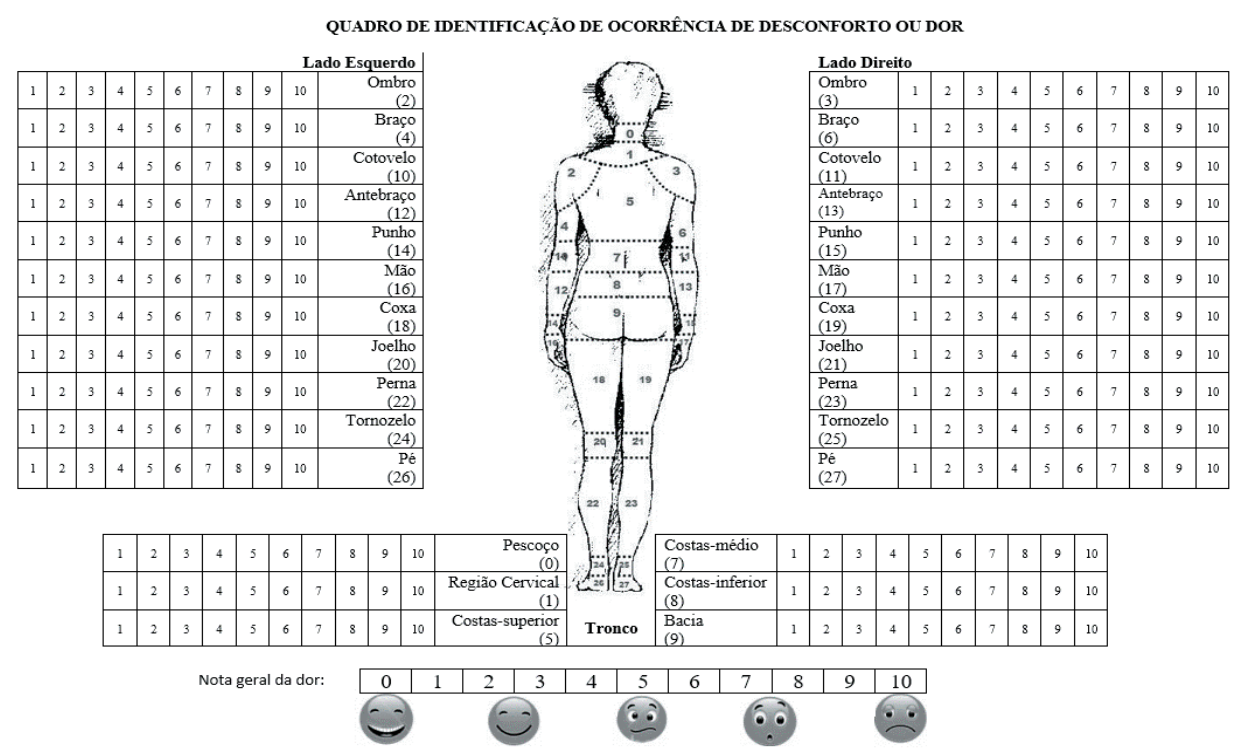

FIGURA 2 - Escala de Borg (CR-10) adaptada para verificação do quadro álgico.

\section{Resultados}

O projeto Viva bem com a coluna que você tem!, tem demonstrado uma opção viável para o tratamento da lombalgia. Diante das altas prevalências de lombalgia ${ }^{2}$, o que ocasiona altas demandas do serviço, o projeto consegue disponibilizar um tratamento coletivo de caráter multidisciplinar, reduzindo a espera ao atendimento ao tratamento convencional individualizado oferecido pela Clínica Municipal de Reabilitação.

Com relação à efetividade, em 2007, foi realizado um estudo quase-experimental com 49 participantes do projeto, com o objetivo de verificar a diminuição do quadro álgico realizado ${ }^{13}$. Para tanto, foi verificado que após 32 sessões de intervenção os valores médios da percepção subjetiva da dor no pré-teste foi de 7,68 $(\mathrm{DP}=0,66)$ e no pós-teste de $6,18(\mathrm{DP}=0,88)$ obtendo significância estatística $(\mathrm{p}<0,0001)^{13}$.

Em 2010, uma pesquisa foi realizada como objeto de uma tese de doutorado. Neste estudo experimental com 109 usuários do Sistema Único de Saúde, onde um grupo realizava apenas tratamento fisioterápico e o outro grupo realizava o Viva bem com a coluna que você tem! ${ }^{14}$. Como resultado do estudo, foi verificado que o grupo que realizava o trabalho multidisciplinar conseguiu uma recuperação em tempo menor, além de melhora significativa nas valências físicas, principalmente na diminuição da dor ${ }^{14}$.

Em Trabalho de Término de Curso em Educação Física, realizado em 2015, buscou-se avaliar elementos de alcance, adoção, efetividade, implementação e manutenção ${ }^{15}$. O estudo envolveu 28 idosos participantes do projeto. Para tanto, foi verificado um alcance de 3,8\% e uma prevalência da participação feminina (72\%). Nível de satisfação foi avaliado entre os usuários obtendo média de 9,2 em uma escala de 0 a $10^{15}$. Além disso, verificou-se melhora na dor e na força de preensão 
manual em 4 meses de intervenção ${ }^{15}$. Por fim, análise qualitativa foi realizada por meio de um grupo focal, onde obteve-se alguns depoimentos como: "não conseguia andar e sentia muita dor, após o projeto as dores sumiram e me sinto mais confiante"; "sinto muita falta das atividades no período de férias, as atividades só me fazem bem"; "me sinto mais forte, e não consigo ficar sem atividades físicas"; "às vezes sinto dores ainda, mas os exercícios ajudam bastante"15, demonstrando, com isso, os benefícios do exercício físico.

Além dos resultados supracitados, a capacitação e treinamento de pessoal é um dos objetivos do projeto. Assim, já atuarem 13 estagiários do Curso de Educação Física e 7 voluntários, os quais produziram juntamente com o grupo de trabalho, 8 apresentações como temas livres em congressos e seminários, sendo premiado em 2005 no II $^{\circ}$ Congresso de Coluna de Santa Catarina.

Apesar da existência do projeto desde 2004 e da inserção da ação como área de atuação do Mafra em Forma da SMS - programa institucionalizado por meio de lei - o desafio atual do "Viva bem com a coluna que você tem!" é a manutenção das ações e a diminuição do tempo de espera para o início das atividades, o que vem ocorrendo mediante espera das assinaturas dos convênios entre as instituições. Além disso, a mudança em cargos de chefia, tanto na Universidade como na SMS, podem influenciar o interesse da continuidade do projeto.

Ainda, percebe-se a necessidade do aumento do alcance, ofertando um número maior de vagas, aumento da frequência semanal e expansão de atividades similares a serem realizadas nas Unidades Básicas de Saúde por meio de parceria com as ações de promoção da saúde já existentes na SMS, como o Núcleo de Apoio à Saúde da Família e o Programa Academia da Saúde.

Observa-se que o projeto, tem constituído com o usuário do Sistema Único de Saúde a melhora/manutenção da aptidão física relacionada à saúde e diminuição do quadro álgico. Com isso, o Viva bem com a coluna que você tem! tem atingido seus resultados e demonstrado que pode ser adotado como uma estratégia alternativa para o tratamento da lombalgia, pois não é invasivo, farmacológico e de alto custo.

\section{Contribuiç̃ões dos autores}

D.R.Petreca (0000-0002-6006-4861) realizou a redação do manuscrito e é responsável por manter o projeto em andamento., F.H.Rodrigues (0000-0001-5725697X) e N.Furquim Junior (0000-0002-6981-100X), foram os idealizadores e aplicadores da intervenção, além de contribuírem com a escrita e levantamento de informações. R.Koaski (0000-0002-1242-480X) e L.E.Becker (0000-0002-24607072 ) contribuíram com a coleta de dados e com a revisão do manuscrito. P.F.Sandreschi (0000-0001-6487-2359) e G.Z.Mazo (0000-0002-7813-5592) contribuíram para a redação e revisão do manuscrito.

\section{Conflito de interesse}

Os autores declaram não haver conflitos de interesse

\section{Referências}

1. Schaafsma FG, Anema JR, van der Beek A. Back pain: Prevention and management in the workplace. Best Pract Res Clin Rheumatol. 2015; 29 (3): 483-94.

2. Meucci RD, Fassa AG, Faria NMX. Prevalence of chronic low back pain: systematic review. Rev Saúde Pública; 2015; 49:73.

3. Hoy D, et al. The global burden of low back pain: estimates from the Global Burden of Disease 2010 study. Ann Rheum Dis. 2014; 73: 968-74. 
4. Melloh M, et al. Differences across health care systems in outcome and cost-utility of surgical and conservative treatment of chronic low back pain: a study protocol. BMC Musculoskelet Disord. 2008; 9 (1):81-9

5. Dagenais $S$, et al. A systematic review of low back pain cost of illness studies in the United States and internationally. Spine J. 2008; 8 (1): 8-20.

6. Searle A, Spink M, Ho A, Chuter V. Exercise interventions for the treatment of chronic low back pain: a systematic review and meta-analysis of randomised controlled trials. Clin Rehabil. 2015; 29(12): 1155-1167.

7. Stieglitz DD, Vinson DR, Hampton MC. Equipment-based Pilates reduces work-related chronic low back pain and disability: A pilot study. J Bodyw Mov Ther. 2016; 20 (1): 74-82.

8. Cuesta-Vargas A, García-Romero JC, Arroyo-Morales M, Diego-Acosta AM, Daly DJ. Exercise, Manual Therapy and Education With or Without High Intensity Deep Water Running for Non-Specific Chronic Low Back Pain: A Pragmatic Randomised Controlled Trial. Am J Phys Med Rehabil. 2011; 90 (7): 526-38.

9. Jorge NSG, Zanin C, Knob B, Wibelinger LM. Physiotherapeutic intervention on chronic lumbar pain impact in the elderly. Rev Dor. 2015;16 (4): 302-5.

10. Knoplich J. Viva bem com a coluna que você tem. 29 ed. São Paulo: Ibrasa, 1997.

11. Borg, G. Borg's perceived exertion and pain scales. 1 ed. Human Kinetics, 1998.

12. Monteiro J, Faísca L, Nunes O, Hipólito J. Questionário de incapacidade de Roland Morris: adaptação e validação para a população portuguesa com lombalgia. Acta Médica Portuguesa. 2010; 23 (5): 761-766.

13. Petreça DR, Rodrigues FH, Benedetti TB. Efeito do exercício físico na diminuição da dor em pacientes portadores de lombalgia do Sistema Único de Saúde de Mafra - SC. Rev Bras Ativ Fís Saúde. 2007;12 (2):151.

14. Furquim N. A ação combinada de exercício físico e fisioterapia na recuperação da lombalgia em indivíduos acima de 60 anos [tese de doutorado]. Porto Alegre: Pontifícia Universidade Católica do Rio Grande do Sul; 2010.

15. Koaski R. Avaliação de um projeto multidisciplinar para o tratamento da lombalgia por meio do modelo RE-AIM [Trabalho de Conclusão de Curso]. Mafra: Universidade do Contestado; 2015.

Endereço para

Correspondência

Daniel Rogério Petreça

profdaniel@unc.br
Av. Presidente Nereu Ramos, 1071

Jardim do Moinho

Mafra - SC - CEP 89300-000

Fone: (47) 3641-5573
Recebido

10/03/2016 $06 / 12 / 2016$ 24/03/2017 29/04/2017 